\title{
Improved Space Vector Modulation Strategy for AC-DC Matrix Converters
}

\author{
Xiao Liu ${ }^{\dagger}$, Qingfan Zhang ${ }^{*}$, Dianli Hou*, and Siyao Wang* \\ $\dot{\dagger}^{*}$ School of Control Science and Engineering, Shandong University, Jinan, China
}

\begin{abstract}
In this paper, an approach to reduce the common-mode voltage and to eliminate narrow pulse for implemented AC-DC matrix converters is presented. An improved space vector modulation (SVM) strategy is developed by replacing the zero space vectors with suitable pairs of active ones. Further, while considering the commutation time, the probability of narrow pulse in the conventional and proposed SVM methods are derived and compared. The advantages of the proposed scheme include: a $50 \%$ reduction in the peak value of the common-mode voltage; improved input and output performances; a reduction in the switching loss by a reduced number of switching commutations and a simplified implementation via software. Experimental results are presented to demonstrate the correctness of the theoretical analysis, as well as the feasibility of the proposed strategy.
\end{abstract}

Key words: Common-mode voltage, Matrix converter, Narrow pulse, Space vector modulation

\section{INTRODUCTION}

AC-DC energy conversion is commonly used in industry applications such as DC motor drives, uninterruptible power supplies (UPS), high-voltage direct current (HVDC) electric power transmission systems, battery energy storage systems (BESS) and so on. Among the many AC-DC converter topologies available, the pulse-width-modulation (PWM) rectifier [1], which can ideally produce a variable ripple-free dc output current while drawing sinusoidal input currents from the supply at unity power factor, attracts the most attention. The main drawback of this topology is its requirement of a bulky set of line inductors for voltage boosting purposes. The size of these inductors would limit its compactness and raise its costs. In addition to the PWM rectifier, the AC-DC matrix converter or matrix rectifier (MR) [2], [3], can also be considered. The major advantage of this converter is its reduced volume and weight due to the absence of bulky energy storage components. Furthermore, the matrix rectifier can generate load waveforms with a wide range magnitude and variable polarity during motoring and regeneration.

Since Donald and Thomas [3] derived the first modulation technique for the MR by utilizing the Venturini switching theory [4], several modulation methods have been proposed [5]-[7]. Among them, space vector modulation (SVM) has

Manuscript received Jan. 15, 2013; revised Apr. 27, 2013

Recommended for publication by Associate Editor Sangshin Kwak

†Corresponding Author: Liu xiao1985@sina.com

Tel: +86-0531-88396974, Shandong University

${ }^{*}$ Sch. of Control Science and Engineering, Shandong University, China been, up to now, a well-known and commonly used modulation method because of its high performance and relative simplicity. Nevertheless, an improperly designed modulation strategy will introduce many problems into the system, including common-mode interference [8], waveform distortion caused by narrow pulses [9], switching losses, etc.

The common-mode voltage (CMV) with a high magnitude, a high frequency and a high variation ratio produced by power converters can cause early motor winding failures and bearing deterioration, reduce the lifetime of the winding insulation, break the motor insulation easily, and cause wideband electromagnetic interference on the output side. Thus it is very important to reduce the CMV or limit it within certain bounds for adjustable speed drive systems or other types of loads.

Several methods to reduce common-mode voltage have been proposed [8], [10]-[14]. However, these methods were established for matrix converters with an AC output. The CMV in MRs and its suppression have not yet been studied thoroughly. Meanwhile, all of these studies were carried out on the basis of ideal conditions, where the commutation process is neglected.

It is common knowledge that current commutation is a critical issue in matrix converters. A multi-step commutation strategy is essential for guaranteeing safe operation in practical applications. Consequently, since a finite commutation time cannot be ignored, an improper modulation strategy will lead to commutation interruptions [15]-[17]. This in turn can result in waveform distortions and even serious commutation failure. Thus an optimized SVM method, which reduces the narrow pulse probability, has been developed for the MR in [17]. 


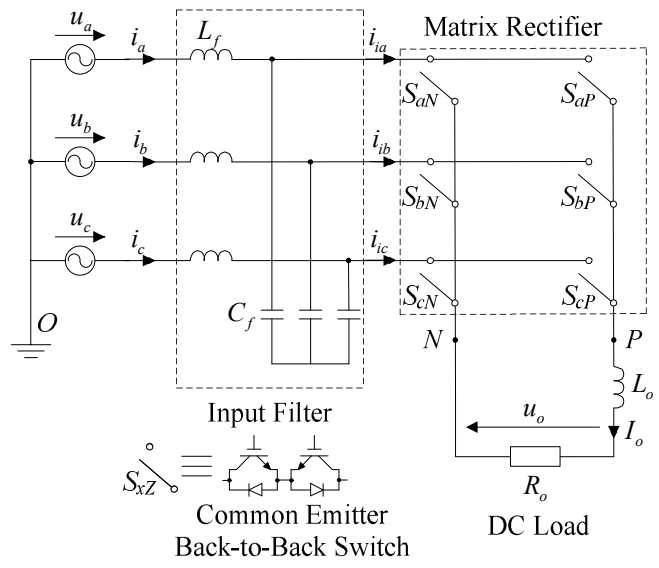

Fig. 1. Matrix rectifier power topology.

However, the number of switching commutations per switching period reaches a maximum of 8 times, which significantly increases the software complexity and the switching loss. In addition, the CMV reduction problem is not covered in [17].

This paper presents a novel SVM method for MRs to reduce the peak value of the CMV perfectly and to improve the quality of the input and output waveforms. In the proposed approach, a group of four active space vectors are used to synthesize the desired output voltage. Although the idea of using a suitable pair of active space vectors instead of zero space vectors was introduced in [8], the narrow pulse probability of this CMV reduction approach is being studied in detail for the first time. An MR prototype is implemented and experiments are carried out with a resistive load. The obtained results verify that a $50 \%$ reduction in the amplitude of the CMV is achieved. They also verify that the performance of the MR is improved and that the output voltage regulation range is expanded by the proposed method.

\section{MATRIX RECTIFIER AND ITS MODULATION STRATEGY}

\section{A. The Conventional SVM Method}

When setting the desired output frequency to 0 , and removing one output leg, the classical three-phase AC-AC matrix converter is reduced to a MR, as illustrated in Fig. 1. The topology consists of a three-phase power source, switching matrix composed by six bidirectional switches, an input LC filter to improve the source current quality and to reduce the source voltage distortion, an output inductor and a resistive load.

The six bidirectional switches in the MR should be commuted under the restriction that only one switch per output phase should be $\mathrm{ON}$ and that it must be $\mathrm{ON}$ at any time instance. This restriction reduces to nine the feasible switching states determining the nine space vectors, as shown in Fig. 2. The subscript in each space vector denotes its corresponding

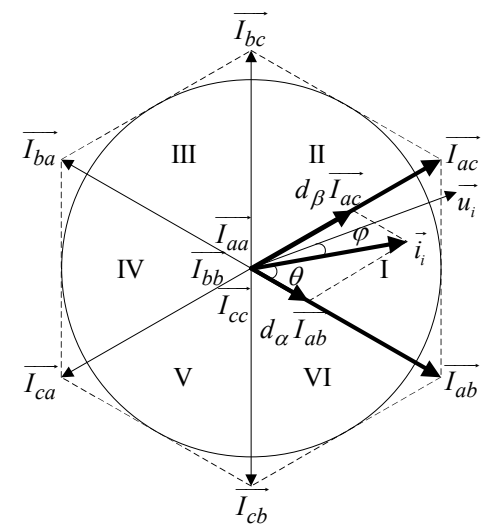

Fig. 2. Space vector diagram in the conventional SVM method.

switching state. For example, $\overrightarrow{I_{a c}}$ denotes switching state $a c$, which connects the input phase $a(c)$ to the output port $P(N)$, like the other eight vectors. The nine space vectors can be divided into two categories: the six active vectors $\overrightarrow{I_{a b}}, \overrightarrow{I_{a c}}$, $\overrightarrow{I_{b c}}, \overrightarrow{I_{b a}}, \overrightarrow{I_{c a}}$ and $\overrightarrow{I_{c b}}$, which yield non-zero output voltages and input currents, and the three zero vectors $\overrightarrow{I_{a a}}, \overrightarrow{I_{b b}}$, and $\overrightarrow{I_{c c}}$.

The conventional SVM algorithm for a MR is explained in [18]-[20]. The space vector hexagon is separated into six sectors by the active vectors. To explain the modulation technique easily without missing the generality of the analysis, referring to Fig. 2, both the input voltage vector $\overrightarrow{u_{i}}$ and the reference input current vector $\vec{I}_{i}$ are assumed to lie in sector I.

In this example, the two active vectors $\overrightarrow{I_{a c}}$ and $\overrightarrow{I_{a b}}$ are used to synthesize the desired input current vector $\vec{I}_{i}$. According to the input current vector synthesis principle, the duty cycles $d_{\alpha}$ and $d_{\beta}$ can be calculated as follows:

$$
\begin{gathered}
d_{\alpha}=T_{\alpha} / T_{s}=m \sin (\pi / 3-\theta) \\
d_{\beta}=T_{\beta} / T_{s}=m \sin \theta
\end{gathered}
$$

where $m$ is the modulation index, $m \in[0,1] ; T_{\alpha}$ and $T_{\beta}$ are the durative times of $\overrightarrow{I_{a b}}$ and $\overrightarrow{I_{a c}}$ in one switching period $T_{s} ; \theta$ is the sector angle defined as the angle between $\vec{I}_{i}$ and its right adjacent active vector $\overrightarrow{I_{a b}} \cdot \theta$ is calculated by:

$$
\theta=f_{\bmod }\left(\omega_{i} t-\varphi, \pi / 3\right)
$$

where $\omega_{i}$ is the angular frequency of the power source; $\varphi$ is the demanded displacement angle of the source currents; $f_{\bmod }(x, y)$ stands for the remainder of $x$ when divided by $y$.

The duty cycle of the zero vector $\overrightarrow{I_{a a}}$ is such that the total duty cycle must be equivalent to the unit at a fixed switching frequency: 


\begin{tabular}{|l|l|l|l|l|l|l|l|l|l|}
\hline $\overrightarrow{I_{b b}}$ & $\overrightarrow{I_{a b}}$ & $\overrightarrow{I_{a a}}$ & $\overrightarrow{I_{a c}}$ & $\overrightarrow{I_{c c}}$ & $\overrightarrow{I_{c c}}$ & $\overrightarrow{I_{a c}}$ & $\overrightarrow{I_{a a}}$ & $\overrightarrow{I_{a b}}$ & $\overrightarrow{I_{b b}}$ \\
\hline$S_{b P}$ & & $S_{a P}$ & $S_{c P}$ & & $S_{a P}$ & $S_{b P}$ \\
\hline$S_{b N}$ & $S_{a N}$ & & $S_{c N}$ & & $S_{a N}$ & $S_{b N}$ \\
\hline$\frac{T_{0}}{8}$ & $\frac{T_{\alpha}}{2}$ & $\frac{T_{0}}{8}$ & $\frac{T_{\beta}}{2}$ & $\frac{T_{0}}{4}$ & $\frac{T_{0}}{4}$ & $\frac{T_{\beta}}{2}$ & $\frac{T_{0}}{8}$ & $\frac{T_{\alpha}}{2}$ & $\frac{T_{0}}{8}$ \\
\hline
\end{tabular}

Fig. 3. Modulation pattern of the conventional SVM method.

$$
d_{0}=T_{0} / T_{s}=1-d_{\alpha}-d_{\beta}
$$

Assuming that the three-phase power source is balanced:

$$
\left\{\begin{array}{l}
u_{a}=U_{s} \sin \left(\omega_{i} t\right) \\
u_{b}=U_{s} \sin \left(\omega_{i} t-2 \pi / 3\right) \\
u_{b}=U_{s} \sin \left(\omega_{i} t+2 \pi / 3\right)
\end{array}\right.
$$

where $U_{s}$ is the amplitude of the input phase voltage. Combining the source voltages defined in (5) and the duty cycles given in (1) and (2), the average value of output voltage and source currents can be obtained in one switching period as:

$$
\begin{gathered}
\overline{u_{o}}=1.5 m U_{\mathrm{s}} \cos \varphi \\
{\left[\begin{array}{l}
\overline{i_{a}} \\
\overline{i_{b}} \\
\overline{i_{c}}
\end{array}\right]=m I_{o}\left[\begin{array}{l}
\sin \left(\omega_{i} t-\varphi\right) \\
\sin \left(\omega_{i} t-\varphi-2 \pi / 3\right) \\
\sin \left(\omega_{i} t-\varphi+2 \pi / 3\right)
\end{array}\right]}
\end{gathered}
$$

(6) and (7) indicate that the maximum output voltage of the $\mathrm{MR}$ is $1.5 U_{s}$ at $m=1$ and $\varphi=0$; the source currents are sinusoidal and the input power factor is fully controllable.

Once the desired output voltage $u_{o}$ and the input power factor angle $\varphi$ are given, the space vectors and their corresponding duty cycles are determined univocally. Then, the modulation pattern is applied to complete the switching period.

\section{B. Modulation Pattern}

Theoretically, the modulation pattern has no impact on the average output voltage or the input currents. However, in practical applications, since the commutation time cannot be ignored, an improperly designed space vector configuration will result in performance deterioration [15], [21]. Among the several modulation patterns available for the conventional SVM method, the double-sided symmetric pattern with three zero vectors has been proven to be optimal from the point of view of minimized narrow pulse probability [17]. With reference to the example case of $\vec{I}_{i}$ lying in sector I and the demanded displacement angle $\varphi=0$, the space vectors selected are $\overrightarrow{I_{a c}}, \overrightarrow{I_{a b}}, \overrightarrow{I_{a a}}, \overrightarrow{I_{b b}}$, and $\overrightarrow{I_{c c}}$, as shown in Fig.

\begin{tabular}{|c|c|}
\hline Space vectors & Peak value of $u_{\mathrm{CMV}}$ \\
\hline $\overrightarrow{I_{a c}}, \overrightarrow{I_{a b}}$ & $\sqrt{3} U_{s} / 4$ \\
\hline $\overrightarrow{I_{b c}}, \overrightarrow{I_{c b}}$ & $-U_{s} / 2$ \\
\hline $\overrightarrow{I_{b a}}, \overrightarrow{I_{c a}}$ & $-\sqrt{3} U_{s} / 4$ \\
\hline $\overrightarrow{I_{a a}}$ & $U_{s}$ \\
\hline $\overrightarrow{I_{b b}}, \overrightarrow{I_{c c}}$ & $-\sqrt{3} U_{s} / 2$ \\
\hline
\end{tabular}
3. As a consequence, 8 switching commutations occur in one switching period.
TABLE I

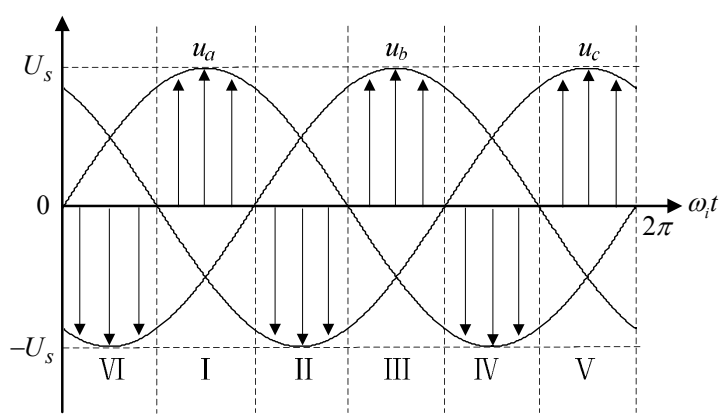

Fig. 4. Distribution pattern of $u_{\mathrm{CMV}}$ with the conventional SVM method.

\section{The Proposed SVm Method to ReducE CMV AND NARROW PULSES}

\section{A. CMV of a $M R$}

When using a MC with an AC output or a MR with a DC output two types of output voltage are generated while working. One is differential-mode voltage, which is available for the load; and the other is common-mode voltage, which is defined with reference to the system neutral point and is detrimental to electrical equipment. The value of CMV can be obtained by dividing the sum of the output phase voltages with the number of output phases. Hence, in a MR, the following is obtained:

$$
u_{C M V}=\left(u_{P O}+u_{N O}\right) / 2
$$

Regardless of the power source, $u_{C M V}$ is determined by the output voltages which depend only on the switching states of the MR. Take $\vec{I}_{i}$ located in sector I for an example. According to (8), the $u_{C M V}$ peak value of all nine feasible space vectors within this sector are summarized in TABLE I.

According to TABLE I, the maximum $u_{C M V}$ is equal to the magnitude of the input phase voltage $U_{s}$ when the zero vector $\overrightarrow{I_{a a}}$ is selected. The same conclusion can be drawn in the other sectors. This state occurs because the zero vector with the maximum $u_{C M V}$ peak value is always selected in each switching period. Subsequently, $u_{C M V}$ traces along the envelope of the input phase voltage with the maximum value, as shown in Fig. 4. 


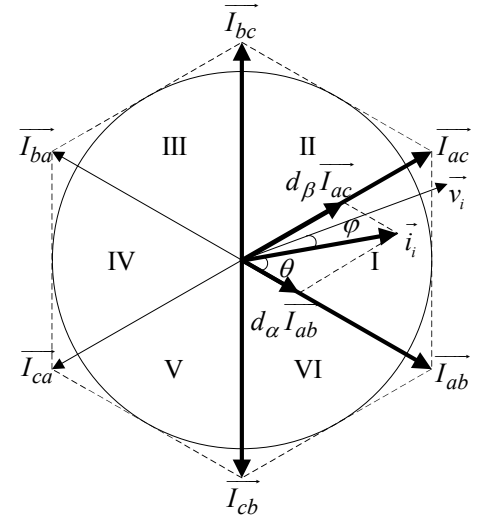

Fig. 5. Space vector diagram in the proposed SVM method.

TABLE II

SWITCHING STATES WITH THE PROPOSED SVM METHOD

\begin{tabular}{ccccc}
\hline \hline Sector & $d_{\alpha}$ & $d_{\beta}$ & $d_{01}$ & $d_{02}$ \\
\hline I & $\overrightarrow{I_{a b}}$ & $\overrightarrow{I_{a c}}$ & $\overrightarrow{I_{b c}}$ & $\overrightarrow{I_{c b}}$ \\
II & $\overrightarrow{I_{a c}}$ & $\overrightarrow{I_{b c}}$ & $\overline{I_{b a}}$ & $\overrightarrow{I_{a b}}$ \\
III & $\overrightarrow{I_{b c}}$ & $\overrightarrow{I_{b a}}$ & $\overrightarrow{I_{c a}}$ & $\overrightarrow{I_{a c}}$ \\
IV & $\overrightarrow{I_{b a}}$ & $\overrightarrow{I_{c a}}$ & $\overrightarrow{I_{c b}}$ & $\overrightarrow{I_{b c}}$ \\
V & $\overrightarrow{I_{c a}}$ & $\overrightarrow{I_{c a}}$ & $\overrightarrow{I_{a b}}$ & $\overrightarrow{I_{b a}}$ \\
VI & $\overrightarrow{I_{c b}}$ & $\overrightarrow{I_{a b}}$ & $\overrightarrow{I_{a c}}$ & $\overrightarrow{I_{c a}}$ \\
\hline \hline
\end{tabular}

\section{B. The proposed SVM method}

In order to mitigate the common-mode voltage in a MR, the switching patterns should not invoke any zero vectors. The proposed SVM method utilizes a group of four active vectors to produce the reference input current vector $\vec{I}_{i}$. These four active vectors are the two active vectors closest to $\vec{I}_{i}$ and its two neighbors. As shown in Fig. 5, in sector 1, the selected active vectors are $\overrightarrow{I_{a b}}, \overrightarrow{I_{a c}}, \overrightarrow{I_{b c}}$ and $\overrightarrow{I_{c b}}$.

The duty cycles for these active vectors are calculated according to the following expression:

$$
\overrightarrow{I_{i}}=d_{\alpha} \overrightarrow{I_{a b}}+d_{\beta} \overrightarrow{I_{a c}}+d_{01} \overrightarrow{I_{b c}}+d_{02} \overrightarrow{I_{c b}}
$$

From (9), the duty cycles become:

$$
\begin{gathered}
d_{\alpha}=T_{\alpha} / T_{s}=m \sin (\pi / 3-\theta) \\
d_{\beta}=T_{\beta} / T_{s}=m \sin \theta \\
d_{01}=d_{02}=T_{01} / T_{s}=T_{02} / T_{s}=\left(1-d_{\alpha}-d_{\beta}\right) / 2
\end{gathered}
$$

Table II shows the space vectors obtained with the proposed SVM method according to the location of the desired input current vector. By using four active vectors to synthesize $\vec{I}_{i}$, the maximum output voltage is unaffected and remains $1.5 U_{s}$.

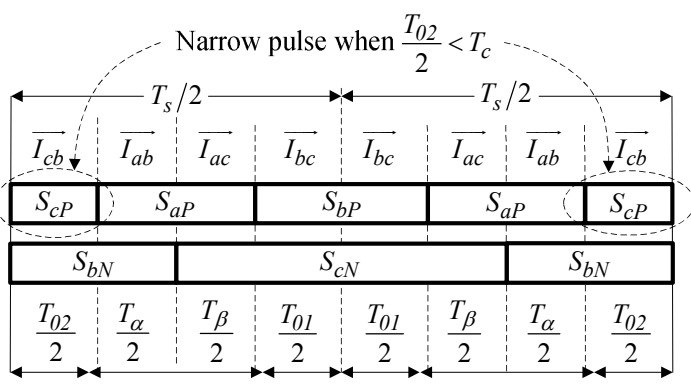

Fig. 6. Modulation pattern of the proposed SVM method.

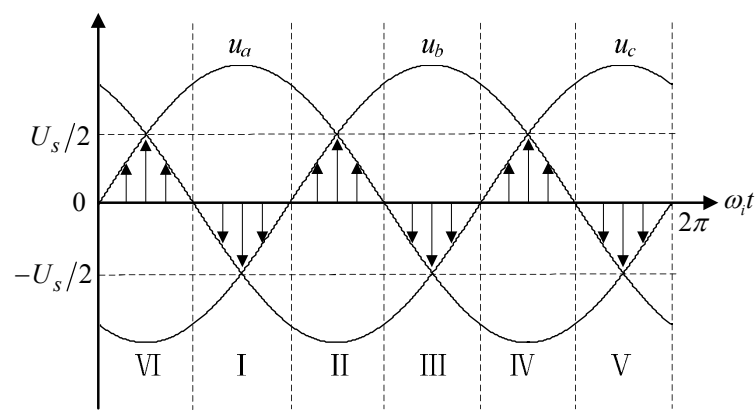

Fig. 7. Distribution pattern of CMV with the proposed method.

\section{Modulation pattern and CMV reduction}

According to the previously explained principle of selecting the active space vectors in the proposed SVM method, the modulation pattern can be arranged symmetrically, as shown in Fig. 6. The number of switching commutations in the proposed method is 6 .

The proposed SVM method discards the zero vectors that produce the maximum $u_{C M V}$ peak value. Instead, a suitable pair of active vectors with the same absolute instantaneous value and opposite polarities is used to accomplish the function of the zero vectors. As a result, the proposed SVM method always generates the smallest $u_{C M V}$ calculated from (8), as shown in Fig. 7. In the proposed SVM method, the amplitude of $u_{C M V}$ is reduced from $U_{s}$ to $U_{s} / 2$ as a result of a $50 \%$ reduction.

Besides the peak value of $u_{C M V}$, its root-mean-square (RMS) value can also be employed to evaluate the common-mode voltage mitigation ability of the proposed method. The RMS value of $u_{C M V}$ generated by the conventional SMV method and the proposed method in the $N$ th switching period can be calculated by:

$$
u_{C M V R M S n}=\sqrt{\frac{1}{T_{S}} \int_{N T_{S}}^{(N+1) T_{S}} u_{C M V} d t}
$$

Fig. 8 shows the curves of $u_{C M V R M S n c}$ and $u_{\text {CMVRMSnp }}$ changing with $m$ and $\theta$ under unity power factor operation. The subscripts $c$ and $p$ stand for the conventional and the proposed SVM methods, respectively. At a certain modulation index, the conventional SMV method shows a decrement in the per-switching period of the RMS value correlated with the increment of the sector angle $\theta$, as shown in Fig. 8(a). On the 


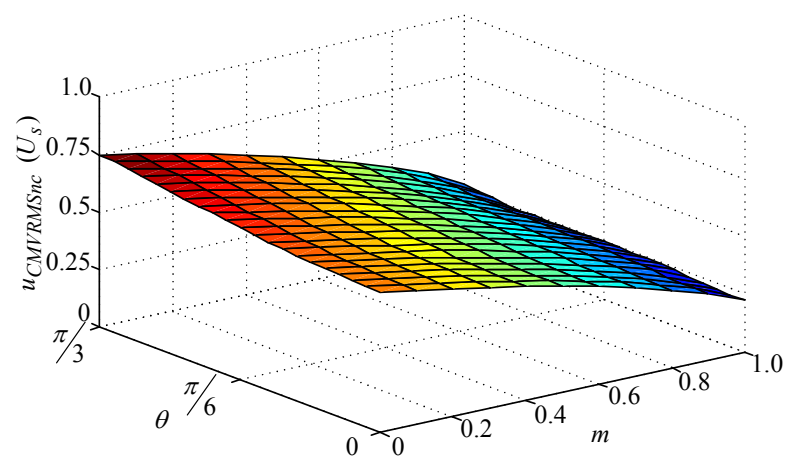

(a)

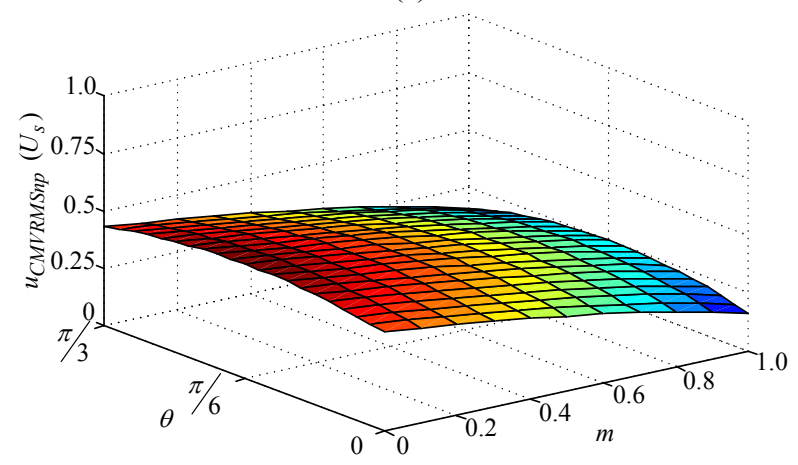

(b)

Fig. 8. Per-switching period RMS value of $u_{\mathrm{CMV}}$ : (a) the conventional method; (b) the proposed method.

other hand, the minimum RMS value of $u_{C M V}$ in the proposed method is obtained in both edges of the sector. When the reference input current vector $\vec{I}_{i}$ moves to the middle of the sector, $u_{\text {CMVRMSnp }}$ reaches its maximum value. At a certain sector angle $\theta$, the RMS values of $u_{C M V}$ in both methods decrease as the modulation index grows. The per-switching period RMS value of $u_{C M V}$ in the proposed method is always smaller than that in the conventional method for any given $m$ and $\theta$ combination.

The per-fundamental period RMS value of $u_{\mathrm{CMV}}$ is obtained by integrating over the entire period as follows:

$$
u_{C M V R M S f}=\sqrt{\frac{1}{2 \pi} \int_{0}^{2 \pi} u_{C M V} d \omega_{i} t}
$$

Fig. 9 shows the waveform of $u_{C M V R M S f}$ as a function of the modulation index. From Fig. 9, a reduction in the per-fundamental period RMS value of $u_{\mathrm{CMV}}$ is achieved by the proposed method. The ratio of decline reaches a maximum value of $28.6 \%$ when $m$ is 0 , and decreases to $20 \% \sim 26 \%$ in the middle area of the modulation range. When the modulation index approaches 1.0, the proportion is at a minimum of $9 \%$.

\section{Narrow pulse probability analysis}

Current commutation is an important issue for the reliable and safe operation of a matrix rectifier. When commutation from one switch to another is required by the modulator, the

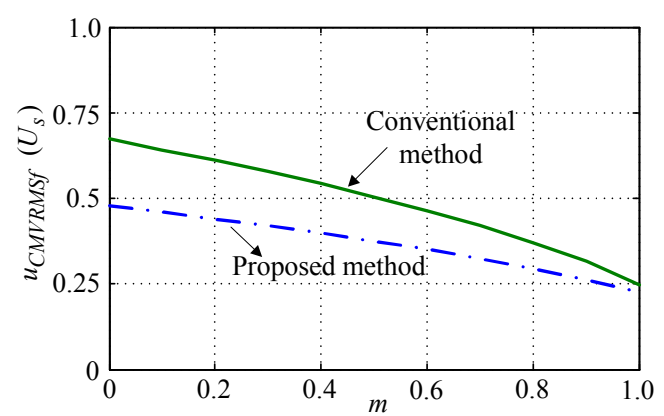

Fig. 9. Per-fundamental period RMS value of $u_{\mathrm{CMV}}$.

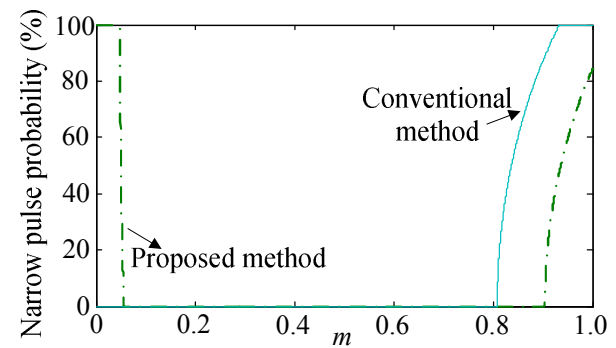

(a)

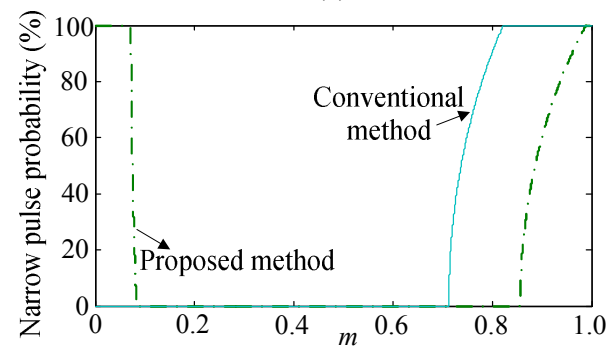

(b)

Fig. 10. Probability of narrow pulses of the three method: (a) $T_{c}=4 \mu \mathrm{s} ;$ (b) $T_{c}=6 \mu \mathrm{s}$.

multi-step commutation strategy needs a finite time to complete the whole sequence. During this time, any new requirement will cause the unfinished process to be interrupted. Hence, the switching state, whose time interval is shorter than the commutation time, is considered to be a narrow pulse. A narrow pulse will result in an incomplete commutation sequence and lead to an output open-circuit. This is not allowed due to the absence of a free-wheeling path in MRs.

Under the narrow pulse condition, two solutions can be considered to achieve a safe commutation. The first one is extending the short durative time of the narrow pulse to the commutation time, so that the commutation process can be accomplish successfully. The second one is neglecting the SVM pulse so the required commutation is canceled. Nevertheless, both approaches have to modify the duty cycles of the space vectors which results in input current distortions and output voltage errors.

Define the commutation time as $T_{c}$ and take the proposed SVM method as an example. The switching state of switch $S_{c P}$ is a narrow pulse when $T_{02} / 2<T_{c}$, as indicated by the dashed ellipses in Fig. 6. Similarly, a narrow pulse will be produced by its corresponding switching state when any of the following 


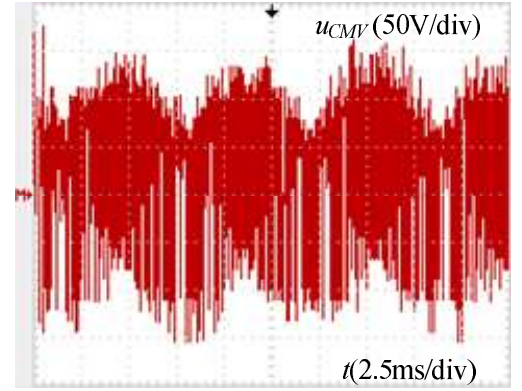

(a)

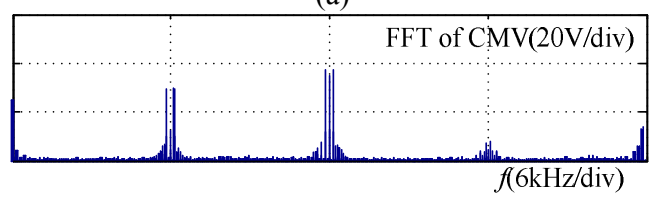

(b)

Fig. 11. Experimental results of $u_{C M V}$ and its FFT with the conventional method: (a) $u_{C M V}$ waveform; (b) FFT of $u_{C M V}$.

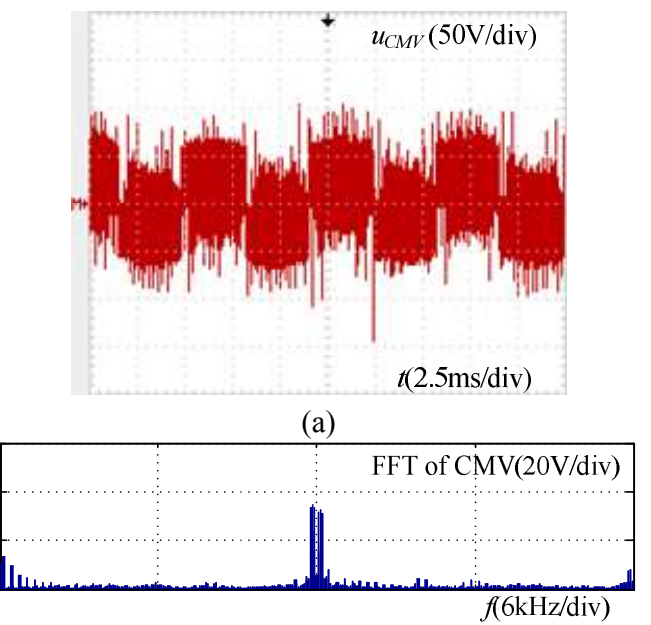

(b)

Fig. 12. Experimental results of $u_{C M V}$ and its FFT with the proposed method: (a) $u_{C M V}$ waveform; (b) FFT of $u_{C M V}$.

conditions is satisfied:

$$
\left\{\begin{array}{c}
T_{02} / 2<T_{c},\left(T_{\alpha}+T_{\beta}\right) / 2<T_{c}, T_{01}<T_{c} \\
\left(T_{02}+T_{\alpha}\right) / 2<T_{c},\left(T_{01}+T_{\beta}\right)<T_{c}
\end{array}\right.
$$

By substituting $T_{01}=T_{02}=T_{0} / 2$ into (15), the following simplified conditions can be obtained:

$$
\left\{\begin{array}{c}
T_{\alpha}+T_{\beta}<2 T_{c} \\
T_{0}<4 T_{c}
\end{array}\right.
$$

According to (16), the narrow pulse probability of the proposed method relates to both the active vector duty cycles and the zero vector duty cycle, while the duty cycles depend on the modulation index $m$ and the sector angle $\theta$. By substituting (10), (11) and (12) into (16), it is possible to obtain the modulation index range where the narrow pulse conditions are satisfied:

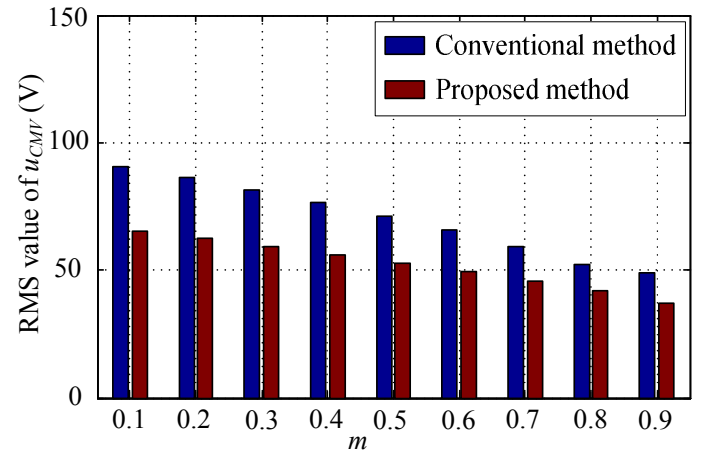

Fig. 13. Experimental results of measurements in the pre-fundamental period RMS value of $u_{C M V}$, with different modulation index.

$$
\begin{gathered}
\text { For } T_{\alpha}+T_{\beta}<2 T_{c}: 0<m<4 T_{\mathrm{c}} / \sqrt{3} T_{\mathrm{s}} \\
\text { For } T_{0}<4 T_{c}: 1-4 T_{\mathrm{c}} / T_{\mathrm{s}}<m<1
\end{gathered}
$$

It is indicated by (17) and (18) that narrow pulses exist only in the very low and very high modulation ranges. When $4 T_{\mathrm{c}} / \sqrt{3} T_{\mathrm{s}}<m<1-4 T_{\mathrm{c}} / T_{\mathrm{s}}$, which is the most common operation condition, the narrow pulses can be eliminated by the proposed SVM method.

In the case of the conventional method, the narrow pulse condition is:

$$
T_{0}<8 T_{c}
$$

Thus the narrow pulse probability of the conventional SVM method only relates to the zero vector duty cycle and can be reduce to 0 when $m<\left(1-8 T_{c} / T_{s}\right)$. However, due to the fact that the zero vector is split into six segments, the duration of each segment becomes very small. This results in a much higher probability in the high modulation area.

In conclusion, the proposed method exhibits the same narrow pulse reduction ability as the conventional method in most of the modulation range. In addition, the proposed method performs even better when the modulation index is high and it only generates more narrow pulses in the very low modulation area.

The narrow pulse probabilities of both modulation strategies are verified preliminarily by the MATLAB software. The obtained simulation results are shown in Fig. 8 with the assumption that the switching period $T_{s}=1 / 6000 \mathrm{~s}$. The commutation time $T_{c}$ is $4 \mu$ s in Fig. 10(a) and $6 \mu$ s in Fig. 10(b).

As seen from Fig. 10, although the narrow pulse probabilities are increased as the commutation time gets longer, the relationship between these two methods remains unchanged and matches the conclusions discussed above. In Fig. 10(a), the narrow pulse probability of the proposed method is 0 when $0.055<m<0.90$. In Fig. $10(\mathrm{~b})$, the narrow pulse probability of the proposed method is 0 when $0.083<m<0.85$. It is clear that the simulation results are in accordance with the theoretical values. 


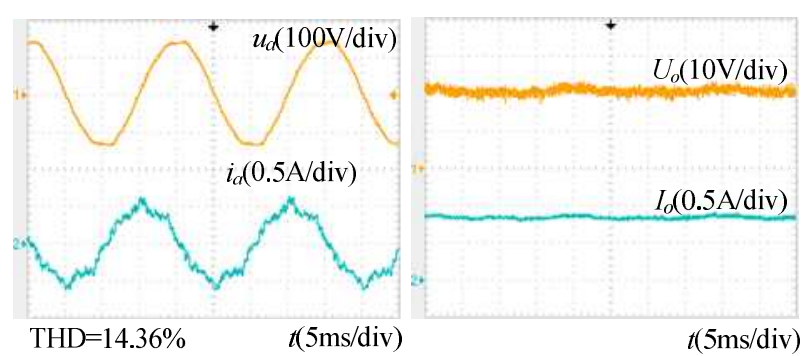

(a)

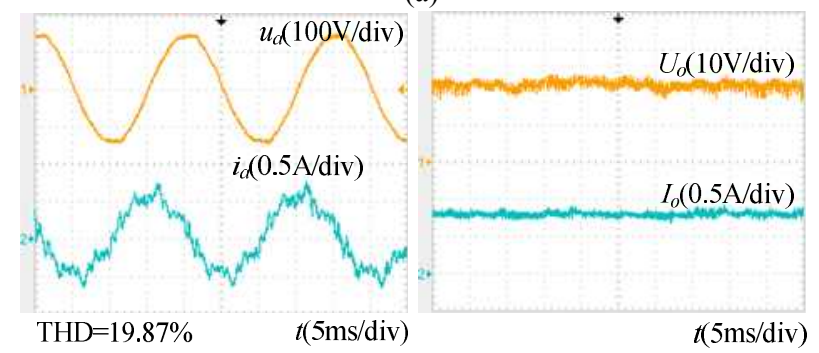

(b)

Fig. 14. Experimental results of input and output waveforms at $m=0.1$ : (a) the conventional method; (b) the proposed method.
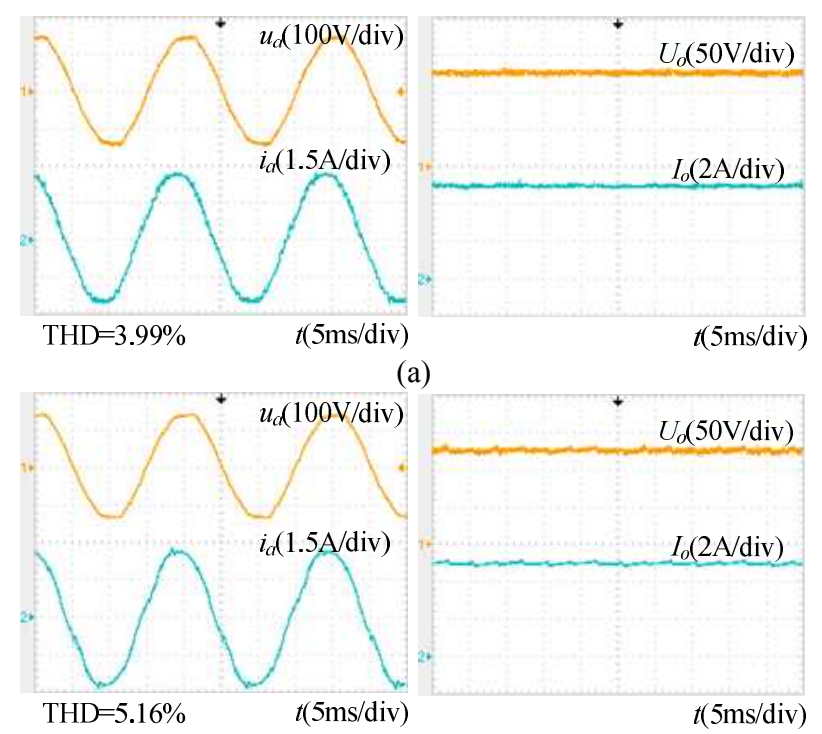

(b)

Fig. 15. Experimental results of input and output waveforms at $m=0.6$ : (a) the conventional method; (b) the proposed method.

\section{EXPERIMENTAL VERIFICATION}

For hardware verification, an experimental prototype is built. The implementation setup consists of a control broad containing a Texas Instruments TMS320F2812 digital signal processor (DSP) and a Xilinx XC9572XL complex programmable logic device (CPLD); an analog broad containing voltage and current sensors; and a power circuit realized by insulated gate bipolar transistors (IGBT). The DSP is used for carrying out the modulation strategies, and the CPLD is used for pulse distribution and carrying out the

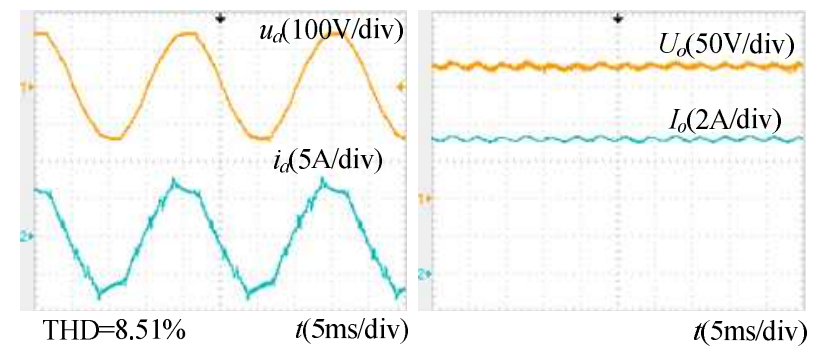

(a)

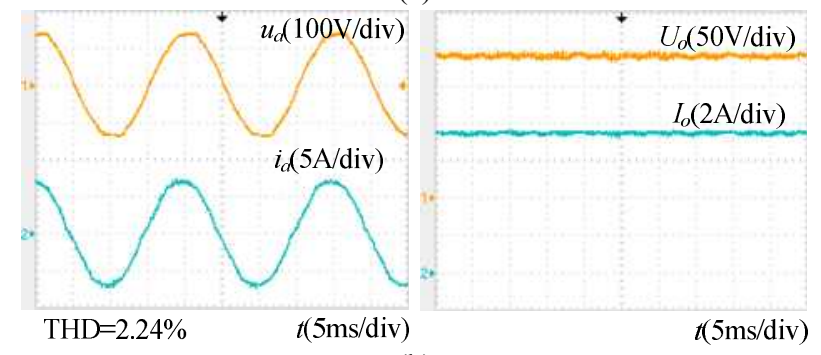

(b)

Fig. 16. Experimental results of input and output waveforms at $m=0.9$ : (a) the conventional method; (b) the proposed method.

current-direction based 4-step commutation [22], [23].

The phase voltage of the power supply is $100 \mathrm{~V} / 50 \mathrm{~Hz}$; the output load is $R_{o}=25 \Omega$; the input filter parameters are $L_{f}=3 \mathrm{mH}$ and $C_{f}=13 \mu \mathrm{F}$ with $27 \Omega$ damping resistors paralleled with the inductors; the output inductor is $50 \mathrm{mH}$; and the switching frequency is $6 \mathrm{kHz}$.

\section{A. The common-mode voltage}

The common-mode voltage waveform and its FFT analysis with the conventional SVM method at $m=0.6$ are shown in Fig. 11. The peak value of $u_{\mathrm{CMV}}$ reaches $141 \mathrm{~V}$ with the corresponding magnitude of the input phase voltage $U_{s}$. Fig. 12 shows the $u_{C M V}$ waveform and its FFT analysis with the proposed method. As shown in Fig. 12, the peak value of $u_{\mathrm{CMV}}$ decreases to $71 \mathrm{~V}$, which means that a $50 \%$ reduction in the amplitude of $u_{C M V}$ is achieved. Furthermore, the FFT analysis of the $u_{C M V}$ waveforms shows the reduced harmonic components for the proposed method. Fig. 13 shows bar graphs of the measurements in the per-fundamental period RMS value of $u_{\mathrm{CMV}}$ using a different modulation index. When compared to the conventional SVM method, the proposed method shows a reduced RMS value of $u_{C M V}$ in the whole modulation range. The experimental results coincide well with the theoretical analysis.

\section{B. The Narrow Pulse}

The input and output performances can be used to evaluate the narrow pulse probability of each modulation strategy. The waveforms in Fig. 14 - Fig. 16 are measured under low, middle and high modulation situations. The extending approach mentioned in the previous section is utilized under the narrow pulse condition.

The input and output waveforms when the modulation index 
$m=0.1$ are shown in Fig. 14. The conventional method shows a better performance with less output current ripple and input current total harmonic distortion. Fig. 15 shows the performance comparison at $m=0.6$. It shows that both of the methods perform well, but that the THD value in the proposed method is slightly larger than that in the conventional method. Fig. 16 shows that at $m=0.9$, the proposed method has a much lower input current THD value and output current ripple. On the other hand, the input and output waveforms with the conventional method are distorted as a result of the much larger narrow pulse probability. Moreover, the output voltage in Fig. 16(a) is lower than the theoretical value, due to a zero vector duty cycle extension to avoid narrow pulses.

\section{CONCLUSIONS}

In this paper, the relationship between the common-mode voltage and the switching states are clarified and an improved SVM method for matrix rectifiers that can reduce the common-mode voltage is developed. Additionally, the narrow pulse probability of the proposed method is discussed in detail with consideration of the commutation time. The proposed modulation method provides several benefits, such as mitigating the uCMV peak value to $50 \%$ with less harmonic components and a reduced RMS value, better waveform qualities of the input and output in the high modulation area, and a reduction of the switching loss due to the ideal switching pattern arrangement. The experimental results verify these advantages, which strengthens the attractiveness of matrix rectifiers for industry applications.

\section{ACKNOWLEDGEMENT}

This paper is financially supported by Science and Technology Development Foundation of Shandong Province, No. 2011GGX10126, and Graduate Independent Innovation Foundation of Shandong University (GIIFSDU), No. YZC10064.

\section{REFERENCES}

[1] J. L. Gao, T. Q. Zheng and F. Lin, "Improved deadbeat current controller with a repetitive-control-based observer for PWM rectifiers," Journal of Power Electronics, Vol. 11, No. 1, pp. 64-73, Jan. 2011.

[2] F. Wen, J. C. Jiang and H. P. Guo, "AC-DC converter based on matrix converter," Trans. of China Electro technical Society, Vol. 24, No. 3, pp. 129-131, Mar. 2009.

[3] D. J. Holmes and T. A. Lipo, "Implementation of a controlled rectifier using AC-AC matrix converter theory," IEEE Trans. Power Electron., Vol. 7, No. 1, pp. 240-249, Jan. 1992.

[4] M. Venturini, "A new sine wave in sine wave out, conversion technique which eliminates reactive elements," in Proc. of Powercon 7, pp. E3/1-E3/15, 1980.
[5] J. Rodriguez, M. Rivera, J. W. Kolar and P. W. Wheeler, "A review of control and modulation methods for matrix converters," IEEE Trans. Ind. Electron., Vol. 59, No.1, pp. 58-70, Jan. 2012.

[6] A. Ishiguro, T. Furuhashi and O. Shigeru, "A novel control method for forced commutated cycloconverters using instantaneous values of input line-to-line voltages," IEEE Trans. Ind. Electron., Vol. 38, No. 3, pp. 166-172, Jun. 1991.

[7] X. H. Yang, X. J. Yang, Z. M. Zhang and J. G. Jiang, "Research on one-cycle control strategy of matrix rectifier," Trans. of China Electro technical Society, Vol. 27, No. 2, pp. 38-44, Feb. 2012.

[8] T. D. Nguyen and H. H. Lee, "Modulation strategies to reduce common-mode voltage for indirect matrix converters," IEEE Trans. Ind. Electron., Vol. 59, No. 1, pp. 129-140, Jan. 2012.

[9] H. Hara, E. Yamamoto and T. Kume, "Improvement of output voltage control performance for low-speed operation of matrix converter," IEEE Trans. Power Electron., Vol. 20, No. 6, pp. 1372-1378, Nov. 2005.

[10] J. C. Han and P. N. Enjeti, "An approach to reduce common-mode voltage in matrix converter," IEEE Trans. Ind. Appl., Vol. 39, No. 4, pp. 1151-1159, Jul. 2003

[11] R. Vargas, U. Ammann, J. Rodriguez and J. Pontt, "Predictive strategy to control common-mode voltage in loads fed by matrix converters," IEEE Trans. Ind. Electron., Vol. 55, No. 12, pp.4372-4380, Dec. 2008.

[12] H. H. Lee and H. M. Nguyen, "An Effective Direct-SVM Method for Matrix Converters Operating with Low Voltage Transfer Ratio," IEEE Trans. Power Electron., Vol. 28, No. 2, pp.920-929, Feb. 2013.

[13] X. Zhang, C. Tong, S. Y. Yang, and Z. Xie, "Restraining common-mode voltage for two-stage matrix converter based on double space vector modulation," China Society for Electrical Engineering, Vol. 30, No. 18, pp. 33-38, Jun. 2010.

[14] R. K. Gupta, K. K. Mohapatra, A. Somani and N. Mohan, "Direct-matrix converter-based drive for a three-phase open-end-winding ac machine with advanced features," IEEE Trans. Ind. Electron., Vol. 57, No. 12, pp. 4032-4042, Dec. 2010.

[15] B. He, H. Lin and H. W. She, "Improvement in output voltage for matrix converter under narrow pulses," China Society for Electrical Engineering, Vol. 29, No. 27, pp. 42-47, Sep. 2009.

[16] X. W. Wang, H. Lin, J. Deng and H. W. She, "Optimization modulation pattern for space vector modulation method of matrix converter in practical application," China Society for Electrical Engineering, Vol. 31, No. 30, pp. 7-15, Oct. 2011.

[17] F. Bo, L. Hua, W. X. Wei and A. Xing, "Optimized Space Vector Modulation Patterns of AC-DC Matrix Converters," China Society for Electrical Engineering, Vol. 32, No. 33, pp. 7-14, Nov. 2012.

[18] D. T. Nguyen, H. H. Lee and T. W. Chun, "A carrier-based pulse width modulation method for indirect matrix converters," Journal of Power Electronics, Vol. 12, No. 3, pp. 448-457, May 2012.

[19] S. Ratanapanachote, J. C. Han and P. N. Enjeti, "A digitally controlled switch mode power supply based on matrix converter," IEEE Trans. Power Electron., Vol. 21, No. 1, pp. 124-130, Jan. 2006.

[20] Y. K. Ping, D. Xiao, M.F. Rahman and M. N. Uddin, "Applying reduced general direct space vector modulation approach of AC-AC matrix converter theory to achieve unity power factor controlled three-phase AC-DC matrix rectifier," 
in Conf. Rec. IEEE IAS, pp. 1-7, 2011

[21] A. Antoni and P. W. Wheeler, "Elimination of waveform distortions in matrix converters using a new dual compensation method," IEEE Trans. Ind. Electron., Vol. 54, No. 4, pp. 2079-2087, Aug. 2007.

[22] H. Wilfried and Z. Marcus, "Multi-Step commutation and control policies for matrix converters," Journal of Power Electronics, Vol. 3, No. 1, pp. 24-32, Jan. 2003.

[23] N. Burany, "Safe control of four-quadrant switches," in Conf. Rec. IEEE IAS, pp. 1190-1194, 1989.

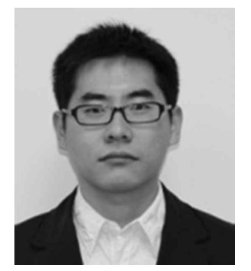

Xiao Liu was born in Jinan, China, in 1985. He received his B.S. and M.S. in Electrical Engineering and Automation from Shanghai Normal University, Shanghai, China, in 2007 and 2010, respectively. $\mathrm{He}$ is currently working toward his Ph.D. in Power Electronics and Electric Drives at Shandong University, Shandong, China. His current research interests include power electronics and control, AC-DC matrix converters, DSP based control applications, and renewable energy.

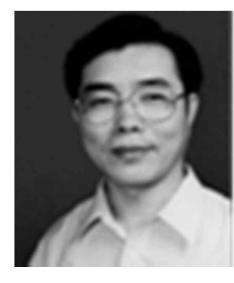

Qingfan Zhang was born in Jining, Shandong, China, in 1949. He is a Professor and Ph.D. supervisor, in the School of Control Science and Engineering, Shandong University, Shandong, China. His current research and teaching interests include power electronics, electronic circuit design, and control systems.

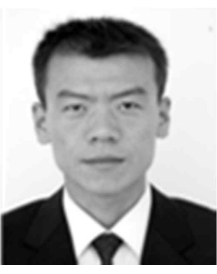

Dianli Hou was born in Liaocheng, Shandong, China, in 1975. He received his M.S. in Circuits and Systems from the Nanjing University of Science and Technology, Nanjing, China, in 2004. Since 2010, he has been working toward his Ph.D. at Shandong University, Shandong, China. $\mathrm{He}$ is currently working as a Teacher in the Department of Electronic and Electrical Engineering, Ludong University, Yantai, China. His current research and teaching interests include power electronics, electronic circuit design, and control systems.

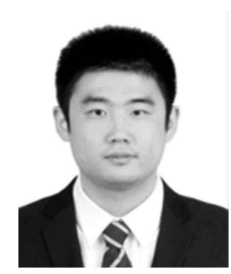

Siyao Wang was born in Jining, Shandong, China, in 1987. He received his B.S. in Control Science and Engineering from Shandong University, Jinan, China, in 2010. Since 2010, he has been working toward his M.S. in Power Electronics and Power Drives at Shandong University. His current research interests include power electronics, energy conversion, and matrix rectifier control and applications. 